\title{
Optical Coherence Tomography based criteria for evaluation of Choriodal Neovascularization
}

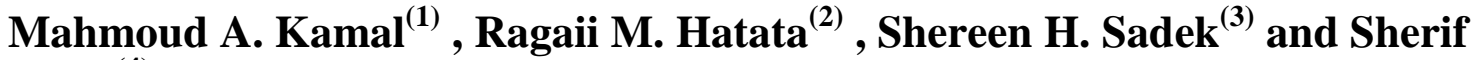 \\ Salah $^{(4)}$
}

(1) Professor of Ophthalmology and Head of ophthalmology department

Faculty of Medicine - Fayoum University

(2) Lecturer of Ophthalmology

Faculty of Medicine - Fayoum University

(3) Lecturer of Ophthalmology

Faculty of Medicine - Fayoum University

(4) Department of ophthalmology

Faculty of Medicine - Fayoum University

Corresponding author: Dr . Sherif Salah

E-mail address: drgehadelhanboli@yahoo.com

Tel:01018337670

Fax: +2 084636583

\section{ABSTRACT}

\section{Purpose:}

To detect the role of optical coherence tomography in evaluation of Choriodal neovascularization in response to treatment with anti vascular endothelial growth factors (VEGF)

\section{Methods:}

A total of 30 eyes with treatment-naïve choriodal neovascular membrane were examined using the OCT system before treatment and after treatment with anti VEGF.

\section{Results:}

In treatment-naïve CNV, The activity of $\mathrm{CNV}$ had been evaluated according to structural OCT by presence of Cystoid Macular Edema (CME), Subretinal Fluid (SRF), Fibrovascular Pigment Epithelial Detachment (FPED) and macular thickening. There is gradual decrease in KEY WORDS : Optical coherence tomography, choriodal neovascularization, anti VEGF,
CMT along the treatment course with repeated intravitreal anti-VEGF injections. $50 \%$ of $\mathrm{CNV}$ patients had $\mathrm{CME}$ at baseline which gradually decreased with repeated anti-VEFG injections and disappeared completely after the third dose, $35 \%$ of CNV patients had SRF at baseline which gradually decreased with repeated antiVEFG injections and disappeared completely after the third dose, Only two patients had PED before treatment, which gradually decreased along the course of treatment by three doses of anti VEGF injections.

\section{Conclusion:}

OCT is an important modality of investigation for diagnosis of $\mathrm{CNV}$ and detecting activity and response with treatment with anti VEGF.

Cystiod macular edema, subretinal fluid, pigment epithelial detachement. 


\section{INTRODUCTION:}

Choriodal neovascularization is associated with abnormal vascular growth from the choroid The neovascular lesion is classified as type 1 choriodal neovascularization $(\mathrm{CNV})$ when expanding through Bruchs membrane (BM) into the space beneath the retinal pigment epithelium (RPE) and as type 2 when spreading into the subretinal space . Abnormal vessel proliferation may also originate from the retinal vasculature and is then refered to as retinal angiomatous proliferation or type 3 CNV (Freund K., et. al.,2011).

Fluorescein angiography (FA) was the gold standard for identifying the neovascular component (Holz F., et. al., 2003 ). Optical coherence tomography (OCT) possibly in combination with FA and indocyanine green (ICGA) should be used to define neovascular disease of the macula .

Optical coherence tomography (OCT) has become an important non-invasive and quick method to diagnose and monitor disease activity during anti vascular endothelial growth factor (VEGF) treatment by revealing sequelae of active CNV such as intra and subretinal fluid (SRF) accumulation and retinal thickenining . On OCT B-scan, CNV appears as subretinal or sub-RPE hyperreflective material .

This study aims to detect the role of OCT in monitoring activity of $\mathrm{CNV}$ along the course of treatment with Anti VEGF.

\section{RESULTS}

The present study evaluated OCT images of thirty patients with choriodal neovascularization due to different etiologies AMD, pathological myopia

\section{PATIENTS AND METHODS}

In our retrospective analysis, patients with all types and different stages of CNV due to different etiologies ( treatment naïve ) were examined by OCT using (Optovue, Fremont, CA, USA). All patients underwent a routine ophthalmic examination including best corrected visual acuity (BCVA) testing using Snellen charts and biomicroscopic examination. Neovascular activity was assessed by SD-OCT imaging by evidence of intra- and/or SRF .At primary presentation, the diagnosis of active $\mathrm{CNV}$ was made by conventional FA. Patients were recruited between August 2017 and February 2018 at the Fayoum University Hospital, Egypt .

Inclusion criteria were patients with choriodal neovascularization due to different etiologies as neovascular AMD , pathological myopia, multifocal chorioditis and trauma, treatment naïve . Patients with previous treatment by Anti VEGF or photodynamic therapy were excluded.

\section{Procedure}

Intra vitreal injection of anti VEGF (Ranibizumab) three successive doses monthly interval.

and multifocal chorioditis. Ten men and twenty women participated in this study with mean age of $50.6 \pm 17.7$ years . 
The mean BCVA was $0.07 \pm 0.06$ decimal.

\section{Correlation between $C N V$ type according to OCT and visual Acuity:}

Five patients have CNV type 1, in which CNV lesion doesn't reach outer retina according to OCT, with median BCVA 0.17 . BCVA improved gradually after injection with Anti VEGF to become 0.2 , 0.2 and 0.25 after first, second and third injection respectively.

Twenty five patients have CNV type 2, in which $\mathrm{CNV}$ lesion reach outer retina according to OCT, with median BCVA 0.06 at base line, which improved gradually after injection with Anti VEGF to become $0.09,0.13$ and 0.14 after first, second and third injection respectively.

There is no significant differences in changes of BCVA between type one and type two CNV (Figure 1).

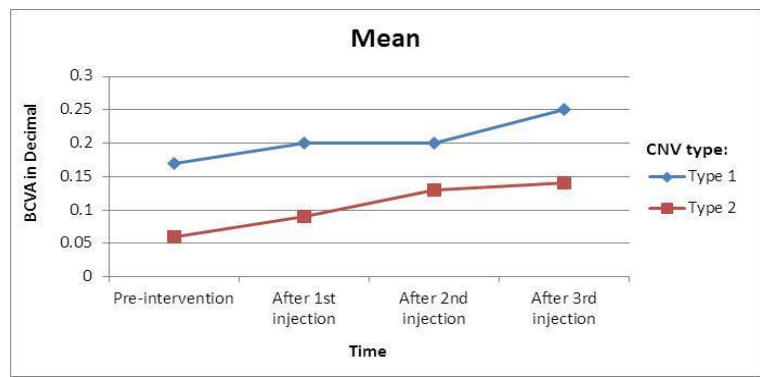

Figure (1): CNV type versus BCVA during follow up period

\section{Changes in Visual Acuity During the course of treatment with Anti VEGF:}

Thirty patients with CNV presented at baseline before treatment, with median BCVA 0.07 (0.01-0.17).

BCVA improve gradually along the course of treatment with Anti VEGF.

After first injection, median BCVA was $0.09(0.01-0.25)$.
After second injection, median BCVA was $0.13(0.03-0.33)$.

After third injection, median BCVA was $0.15(0.30-0.33)$.

4Changes of structural OCT during the course of treatment

The activity of CNV had been evaluated according to structural OCT by presence of Cystoid Macular Edema (CME), Subretinal Fluid (SRF), Fibrovascular Pigment Epithelial Detachment (FPED) and macular thickening .

a.

differences in Central macular thickness (CMT) at different times:

There is gradual decrease in CMT along the treatment course with repeated intravitreal anti-VEGF injections. CMT is a good prognostic factor for improvement of BCVA after repeated anti-VEGF injections. The lower CMT, the better improvement in BCVA. $b$.

ifferences in CME at different times: $50 \%$ of CNV patients had CME at baseline which gradually decreased with repeated anti-VEFG injections and disappeared completely after the third dose (figure 2). 


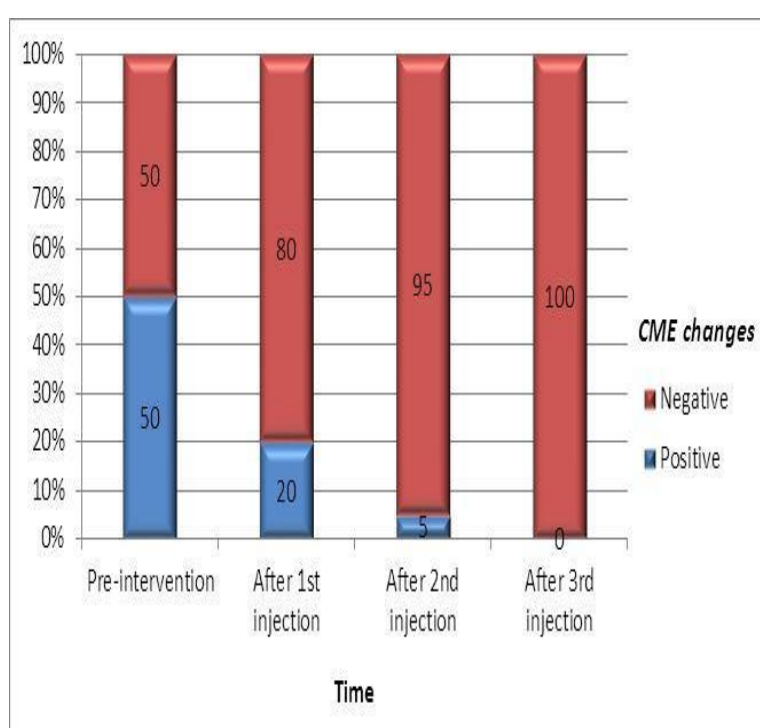

\section{Figure (2): CME changes pre- and post} treatment

CME is considered a positive marker for therapeutic response to anti VEGF treatment, but a poor prognostic factor to improvement in BCVA.

$c$. differences in SRF at different times: $35 \%$ of CNV patients had SRF at baseline which gradually decreased with repeated anti-VEFG injections and disappeared completely after the third dose (Figure 3).

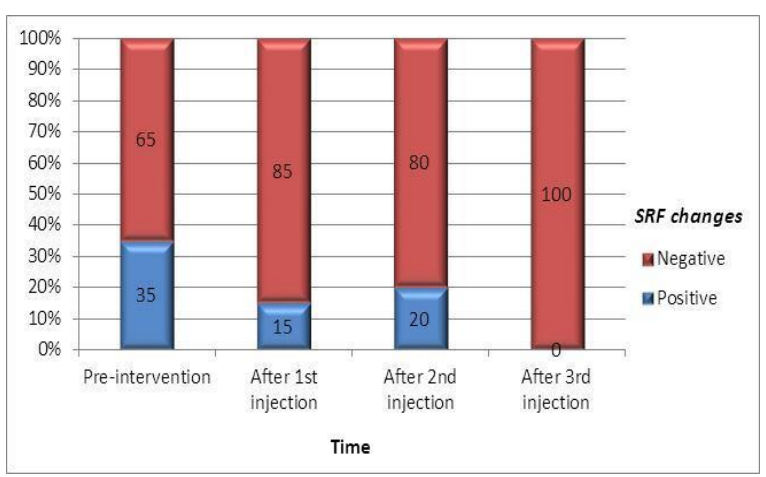

Figure (3): SRF changes pre- and post treatment

SRF is considered a positive marker for therapeutic response to anti VEGF treatment, but a poor prognostic factor to improvement in BCVA in response to anti VEGF.

$d$.

\section{Differences in Pigment Epithelial}

Detachement (PED) at different times:

Only two patients had PED before treatment, which gradually decreased along the course of treatment by three doses of anti VEGF injections.

In our study 25 eyes show gradual regression in response to Anti VEGF along the course of injection (three times). Only five eyes show regression in activity after first injection and become stationary after second and third injection.

change, causing severe vision loss as part of the pathogenesis of several diseases (Gehrs K., et.al., 2006). Structural OCT has become an indispensable tool in the management of Choriodal neovascularization. It allows the clinician to assess fluid exudation from $\mathrm{CNV}$ as manifested by intraretinal cysts, retinal thickening, and subretinal 
fluid accumulation. It identifies the alterations in normal retinal anatomy that are associated with visual potential.

(Giovannini A., et.al., 1999).

Therefore FA or ICGA are still needed in the initial diagnosis of neovascular AMD. Because FA and ICGA require intravenous dye injection, nausea and vomiting are common adverse reactions, and serious anaphylactic reactions are possible. Therefore it is highly desirable to develop a noninvasive method of imaging CNV (Stanga P., et.al., 2003), (Lopez-Saez M., et.al., 1998).

In our study, 25 eyes with active CNV become inactive after three successive doses of anti VEGF, only five eyes become inactive after one dose of anti VEGF.

We evaluated patients with CNV due to different etiologies as AMD, myopia and multifocal chorioditis and were classified by OCT into type 1 and type 2 ,there were only five patients had type 1 (the lesion was at level of choriocapillaries) and 25 patients had type 2 (the lesion reached the outer retina).

There were no significant difference between CNV type and BCVA in response to anti VEGF treatment, as all patients with different $\mathrm{CNV}$ types gradually improved in BCVA in response to treatment.

Morphologic and functionally relevant sequelae for $\mathrm{CNV}$ activity include the following: intraretinal cystoid oedema, SRF, diffuse thickening of the fovea, expanding serous PED, new sub- or intraretinal haemorrhage and BCVA loss attributable to other signs of lesion activity (Tam J., et al., 2011).

Absence of ELM visibility is a more reliable parameter for evaluating $\mathrm{CNV}$ activity than intraretinal/subretinal fluid collection and may constitute a useful option in diagnosing and monitoring the myopic CNV during anti-vascular endothelial growth factor therapy

(Battaglia Parodi M., et.al., 2016). In our study, we evaluated CNV activity by OCT according to CME, SRF,PED and retinal thickening . $50 \%$ of patients had CME before treatment which disappeared after treatment those patients respond well to antiVEGF treatment but with low BCVA. $35 \%$ of patients had SRF at baseline which disappeared after treatment, those patients also had good response to antiVEGF treatment but had poor improvement in BCVA. Only two patients had PED before treatment which disappeared after treatment. Advanced image analyses and structure/function correlation indicated a strong impact of fluid localization within different compartments of the retina. Intraretinal cysts (IRC) were consistently associated with the lowest BCVA values, whereas SRF as individual finding demonstrated superior BCVA. (Spaide R., et al., 2015).

In our study, we observed that patients had intraretinal cysts or subretinal fluid presented with the lowest BCVA which considered poor prognostic factors for improvement in BCVA in response to treatment.

Ranibizumab therapy was effective in improving and maintaining visual acuity in myopic choroidal neovascularization. Early diagnosis and better baseline BCVA were strongly associated with better functional outcomes. Moreover, CNV distinguished by its small size and low CMT responded more favorably, achieving a better visual outcome

(Iacono P., et.al., 2017).

We agreed to Iacono P., et.al., as patients with low CMT had better BCVA at baseline and they respond well to antiVEGF treatment and had better visual outcome. 


\section{REFERENCES:}

[1] Battaglia Parodi M., Iacono P., Bandello F. (2016): Correspondence of leakage of Fluorecein Angiography and Optical Coherence Tomography parameters in diagnosis and monitoring myopic choroidal neovascularization treated by Bevacizumab, Retina; 36(1):104-109.

\section{[2] Freund K., Zweifel S., Engelbert}

M. (2011): Do we need a new classification for choroidal neovascularization in age-related macular degeneration?, Journal of Retina; 30:1333-1349.

[3] Gehrs K., Anderson D., Johnson L., Hageman G. (2006): Age-related macular degeneration - emerging pathogenetic and therapeutic concepts, Annals of Medicine;38(7):450-471.

\section{[4] Giovannini A, Amato GP,} Mariotti C, Scassellati-Sforzolini B. (1999): OCT imaging of choroidal neovascularisation and its role in the determination of patients' eligibility for surgery, British Journal of Ophthalmology; 83:438-42.

[5] Holz FG, Jorzik J, Schutt F, Flach U \& Unnebrink K (2003): Agreement among ophthalmologists in evaluating fluorescein angiograms in patients with neovascular age-related macular degeneration for photodynamic therapy eligibility (FLAP-Study).

Ophthalmology 110: 400-405.
[6] Iacono P., Battaglia Parodi M., Selvi F., Parravano M., Chiaravalloti A., Varano M., Bandello F. (2017): Factors influencing visual acuity in patients receiving anti vascular endothelial growth factor for myopic choroidal neovascularization, Retina; 37(10):1931-1941.

\section{[7] Jian L., Panpan Y., Wen X.} (2013): Current choroidal neovascularization treatment. Ophthalmologica; 230(2):55-61.

[8] Lopez-Saez M., Ordogui E., Tornero P., Baeza A., Sainza T., Zubeldia J., Baeza M. (1998):

Fluorescein-induced allergic reaction, Annals of allergy, asthma and immuonology; 81(5):428-430.

\section{[9] Spaide R., Fujimoto J. and}

Waheed N. (2015): Image artifacts in optical coherence tomography angiography. Retina; 35: 2163-2180.

[10] Stanga P., Lim J., Hamilton P. (2003): Indocyanine green angiography in chorioretinal diseases: indications and interpretation: an evidence-based update, Ophthalmology; 110:15-21.

\section{[11] Tam J, Tiruveedhula P \&}

Roorda A (2011): Characterization of single-file flow through human retinal parafoveal capillaries using an adaptive optics scanning laser ophthalmoscope. Biomedical Optics Express 2: 781-793. 